\title{
Collective mechanism of dilepton production in high-energy nuclear collisions
}

\author{
I.N. Mishustin ${ }^{a, b}$, L.M. Satarov ${ }^{a}$, H. Stöcker ${ }^{c}$ and W. Greiner \\ a The Kurchatov Institute, 123182 Moscow, Russia \\ ${ }^{b}$ The Niels Bohr Institute, DK-2100 Copenhagen Ø, Denmark \\ ${ }^{c}$ Institut für Theoretische Physik, J.W. Goethe Universität, \\ D-60054 Frankfurt am Main, Germany
}

\begin{abstract}
Collective bremsstrahlung of vector meson fields in relativistic nuclear collisions is studied within the time-dependent Walecka model. Mutual deceleration of the colliding nuclei is described by introducing the effective stopping time and average rapidity loss of baryons. It is shown that electromagnetic decays of virtual $\omega$-mesons produced by bremsstrahlung mechanism can provide a substantial contribution to the soft dilepton yield at the SPS bombarding energies. In particular, it may be responsible for the dilepton enhancement observed in 160 AGev central $\mathrm{Pb}+\mathrm{Au}$ collisions. Suggestions for future experiments to estimate the relative contribution of the collective mechanism are given.
\end{abstract}

A high degree of baryon stopping observed in relativistic nuclear collisions implies a strong space-time variation of the collective baryon current in these reactions. According to the timedependent Walecka model [1], in this situation it is natural to expect the excitation of propagating waves (bremsstrahlung) of classical meson fields. Conversion of virtual mesons into real particles may provide an additional important source of secondary particles in nuclear collisions. Unlike the conventional mechanism of incoherent hadron-hadron collisions the contribution of this source increases strongly (quadratically) with the number of participating nucleons. Following this idea in Refs. [2-4] we considered the production of pions, baryon-antibaryon pairs and dileptons by the collective bremsstrahlung and decay of virtual $\omega$-mesons. It was shown that the role of the Collective Bremsstrahlung Mechanism (CBM) grows with bombarding energy and that it becomes important in central collisions of heavy nuclei already at the SPS energies.

The study of dilepton production is especially interesting because of the strong enhancement of soft dileptons observed in nuclear collisions by the CERES [5] and HELIOS-3 [6] collaborations. It was shown [7-11] that the contribution of $\pi \pi \rightarrow \rho \rightarrow l^{+} l^{-}$processes may explain the enhancement only if one assumes a strong modification of $\rho$-mesons at intermediate stages of 
a heavy-ion collision. On the other hand, recent calculations of the $\rho$-meson spectral function show 110, 11] that the $\rho$ peak exhibits a substantial broadening in nuclear matter. In this case it is questionable if the $\rho$-meson can be treated as a well-defined quasiparticle. Below we show that the observed enhancement of the dilepton yield may be explained, at least partly, by the contribution of the CBM.

The detailed formulation of the dilepton production within the CBM is described in Ref. [四]. Below we outline only the main points of the model. The 4-momentum distribution of primordial $\omega$-mesons produced by the bremsstrahlung mechanism is determined by the Fourier transform of the collective baryonic current $J_{\mu}(p)$ :

$$
\frac{\mathrm{d}^{4} N_{\omega}}{\mathrm{d}^{4} p}=\frac{g_{V}^{2}}{16 \pi^{3}}\left|J_{\mu}^{*}(p) J^{\mu}(p)\right| \rho_{\omega}\left(p^{2}\right),
$$

where $\rho_{\omega}\left(p^{2}\right)$ is the spectral function of virtual $\omega$ 's describing their deviation from the mass shell $p^{2}=m_{\omega}^{2}$. This spectral function is written in the Breit-Wigner form 田 with the mass $m_{\omega}$ and width $\Gamma_{\omega}$.

The mass distribution of dileptons produced by the CBM can be written as a convolution of the virtual $\omega$ meson spectrum and the differential branching of the $\omega \rightarrow l^{+} l^{-} X$ decay ( $X$ denotes any particle emitted together with the lepton pair):

$$
\frac{\mathrm{d} N_{l^{+} l^{-}}}{\mathrm{d} M}=\int \mathrm{d}^{4} p \frac{\mathrm{d}^{4} N_{\omega}}{\mathrm{d}^{4} p} \cdot \frac{\mathrm{d} B_{\omega \rightarrow l^{+} l^{-} X}}{\mathrm{~d} M}
$$

where $M$ is the dilepton invariant mass. The procedure of calculating the partial widths of the off-mass-shell $\omega$-mesons is described in Ref. [4]. In our calculations we take into account the direct $\left(\omega \rightarrow l^{+} l^{-}\right)$as well as the Dalitz $\left(\omega \rightarrow \pi^{0} l^{+} l^{-}\right)$decay channels.

According to Eq. (四) the collective bremsstrahlung may be important if the Fourier-transformed baryonic current is nonzero in the time-like region $p^{2} \sim M^{2}$, i.e. if the projectile-target mutual deceleration is strong enough. Simple estimates show [2] that noticeable effects may be expected if the effective deceleration time $\tau_{d}$ is shorter than $\hbar / M$. To calculate $J^{\mu}(p)$ we adopt the schematic picture of a heavy-ion collision suggested in Ref. [12]. We consider central collisions of equal nuclei in the $\mathrm{cm}$ frame and assume that $\tau_{d}$ is equal to the passage time, $\tau_{d}=2 R / \sinh y_{0}$, where $R$ is the geometrical radius of the colliding nuclei and $y_{0}$ is the 
initial $\mathrm{cm}$ rapidity. In our dynamical picture the effects of internal compression and transverse motion are disregarded. The interpenetrating nuclei move as a whole along the beam axis with instantaneous velocities $\pm \dot{z}(t)$ which are parametrized as

$$
\dot{z}(t)=v_{f}+\frac{v_{0}-v_{f}}{1+\mathrm{e}^{2 t / \tau_{d}}} .
$$

Here $v_{0}=\tanh y_{0}$ and $v_{f}$ is the final velocity of nuclei at $t \rightarrow+\infty$. The degree of baryonic stopping is characterized by the average rapidity loss $\delta y=y_{0}-\operatorname{Artanh} v_{f}$. For central $\mathrm{Au}+\mathrm{Au}$ collisions at SPS energies we take the value $\delta y=2.4$ motivated by RQMD calculations [2]. The time integrals determining $J^{\mu}(p)$ within this dynamical model are calculated numerically [4] assuming the Woods-Saxon density distribution in the colliding nuclei. In all calculations we take $g_{V}=13.78$.

The resulting mass spectrum of $e^{+} e^{-}$pairs produced in central $160 \mathrm{AGeV} \mathrm{Au+Au} \mathrm{collisions}$ is shown in Fig. 1. The calculations were made assuming that the on-mass-shell $\omega$-mesons have the vacuum mass, $m_{\omega}=0.782 \mathrm{GeV}$ and decay width, $\Gamma_{\omega}=\Gamma_{0}=8.4 \mathrm{MeV}$. We take into account the same acceptance cuts as used in the CERES measurements of the dilepton yield in $\mathrm{Pb}+\mathrm{Au}$ collisions [5]. The jump of the predicted spectrum at $M=0.35 \mathrm{GeV}$ is caused by the cut at low transverse momenta of leptons. Note that the mass resolution corrections are not included in this calculation. One can see that the CBM contribution is important in the same region of intermediate masses, $M=0.35-0.8 \mathrm{GeV}$, where the enhanced dilepton production is observed experimentally.

Of course, dileptons are also produced by the incoherent formation and decay of pions and hadronic resonances. This hadronic "cocktail" contribution has been obtained [5] by the Monte Carlo simulation which includes decays of pions and known meson resonances. The relative abundances of resonances have been found by scaling the pp and pA data. According to Fig. 1, the incoherent dilepton source is more important outside the intermediate mass region. The sum of the CBM and cocktail contributions satisfactorily reproduces the CERES data. However, the data are still slightly underestimated at $M \simeq 0.5-0.6 \mathrm{GeV}$ and a too sharp peak is predicted at $M \simeq m_{\omega}$.

As shown in Refs. [13, 14] the $\omega$-meson width may increase significantly in dense baryonic 
matter. At baryon densities $n \sim n_{0}$, where $n_{0}$ is the normal nuclear density, the in-medium width of $\omega$-mesons may exceed $\Gamma_{0}$ by an order of magnitude. To estimate the effects of the in-medium broadening of virtual $\omega$-mesons, we performed the same calculation as above, but replacing $\Gamma_{0}$ by a higher width $\Gamma_{\omega}=5 \Gamma_{0}$. Fig. 2 shows that the agreement with the data becomes much better in this case.

Spectra of primordial $\omega$ 's produced by the CBM have two characteristic features [2]. First their $p_{T}$ distributions are relatively soft due to the cutoff $p_{T} \lesssim \hbar / R$ imposed by the nuclear form factor. Second, the rapidity spectra of these $\omega$-mesons have two side maxima, symmetrical with respect to $y_{\text {c.m. }}=0$. Hopefully, these features can be seen in the distributions over the total momentum of dileptons. Such measurements can be used in future experiments to extract the collective component above the "background" of the incoherent production.

To illustrate this model prediction we calculated the distributions over the total rapidity of a dilepton pair produced by the CBM in the central $\mathrm{Au}+\mathrm{Au}$ collision at $160 \mathrm{AGeV}$. The results are shown in fig. 3. Unlike the preceding figures, no acceptance cuts are included in this calculation. A strong mass dependence of the dilepton yield, more pronounced at small total rapidities in the $\mathrm{cm}$ frame, is clearly seen. Also one can see the dip at $y_{c . m .}=0$ which is the characteristic signature of the CBM [2]. The dip is partly filled up due to presence of Dalitz decays. Nevertheless, it is well seen, especially at higher masses where the Dalitz contribution is relatively small.

In conclusion, we have shown that the CBM may provide an important source of dileptons in high-energy nuclear collisions. This mechanism can be responsible, at least partly, for the enhanced yield of dileptons observed at the SPS bombarding energies. In order to observe the collective bremsstrahlung contribution it is highly desirable to extend the experimental acceptance to lower $p_{T}$, to improve the mass resolution and to measure the rapidity dependence of the dilepton yield.

The authors thank for the support from Graduirtenkolleg (Universität Frankfurt), DFG, BMFT and the Carlsberg Foundation. 


\section{References}

[1] B.D. Serot and J.D. Walecka, Adv. in Nucl. Phys. 16, 1 (1985).

[2] I.N. Mishustin, L.M. Satarov, H. Stöcker, and W. Greiner, Phys. Rev. C 52, 3315 (1995).

[3] I.N. Mishustin, L.M. Satarov, and H. Stöcker, in Proc. Int. Conf. Nuclear Physics at the Turn of the Millenium (Wilderness, South Africa, 1996), eds. H. Stöcker, A. Gallman, J.H. Hamilton, World Scientific, 1997, p. 522;

I.N. Mishustin, L.M. Satarov, H. Stöcker, and W. Greiner, hep-ph/9611295.

[4] I.N. Mishustin, L.M. Satarov, H. Stöcker, and W. Greiner, hep-ph/9709384.

[5] G. Agakishiev et al. (CERES Collab.), Phys. Rev. Lett. 75, 1272 (1995); Nucl. Phys. A610, 317c (1996).

[6] M. Masera (HELIOS-3 Collab.), Nucl. Phys. A590, 93c (1995).

[7] G.Q. Li, C.M. Ko, and G.E. Brown, Phys. Rev. Lett. 75, 4007 (1995); C.M. Ko, G.Q. Li, G.E. Brown, and H. Sorge, Nucl. Phys. A610, 342c (1996).

[8] W. Cassing, W. Ehehalt, and C.M. Ko, Phys. Lett. B363, 35 (1995).

[9] L.A. Winckelmann et al., Nucl. Phys. A610, 116c (1996).

[10] R. Rapp, G. Chanfray, and J. Wambach, hep-ph/9702210.

[11] W. Cassing, E.L. Bratkovskaya, R. Rapp, and J. Wambach, nucl-th/9708020.

[12] D. Vasak, H. Stöcker, B. Müller, and W. Greiner, Phys. Lett. 93B, 243 (1980); D. Vasak, B. Müller, and W. Greiner, Phys. Scr. 22, 25 (1980).

[13] G. Wolf, B. Friman, and M. Soyer, nucl-th/970755.

[14] F. Klingl, N. Kaiser, and W. Weise, hep-ph/9704398, to be publ. in Nucl. Phys. A 


\section{Figure captions}

Fig. 1. Invariant mass distributions of $e^{+} e^{-}$pairs in central $160 \mathrm{AGeV} \mathrm{Au+Au} \mathrm{collisions.}$ The collective bremsstrahlung contribution is shown by the dashed line. The dotted line is the result of Monte-Carlo simulation (hadronic cocktail) from Ref. [5]. The solid curve gives the sum of these two mechanisms. Preliminary experimental data for central $\mathrm{Pb}+\mathrm{Au}$ collisions are taken from Ref. [5].

Fig. 2. The same as Fig. 1, but with the dashed line calculated for the increased $\omega$-meson width $\Gamma_{\omega}=5 \Gamma_{0}=42 \mathrm{MeV}$.

Fig. 3. Total rapidity distribution of $e^{+} e^{-}$pairs produced by bremsstrahlung mechanism at different invariant masses $M$. No acceptance cuts are included in the calculation. 


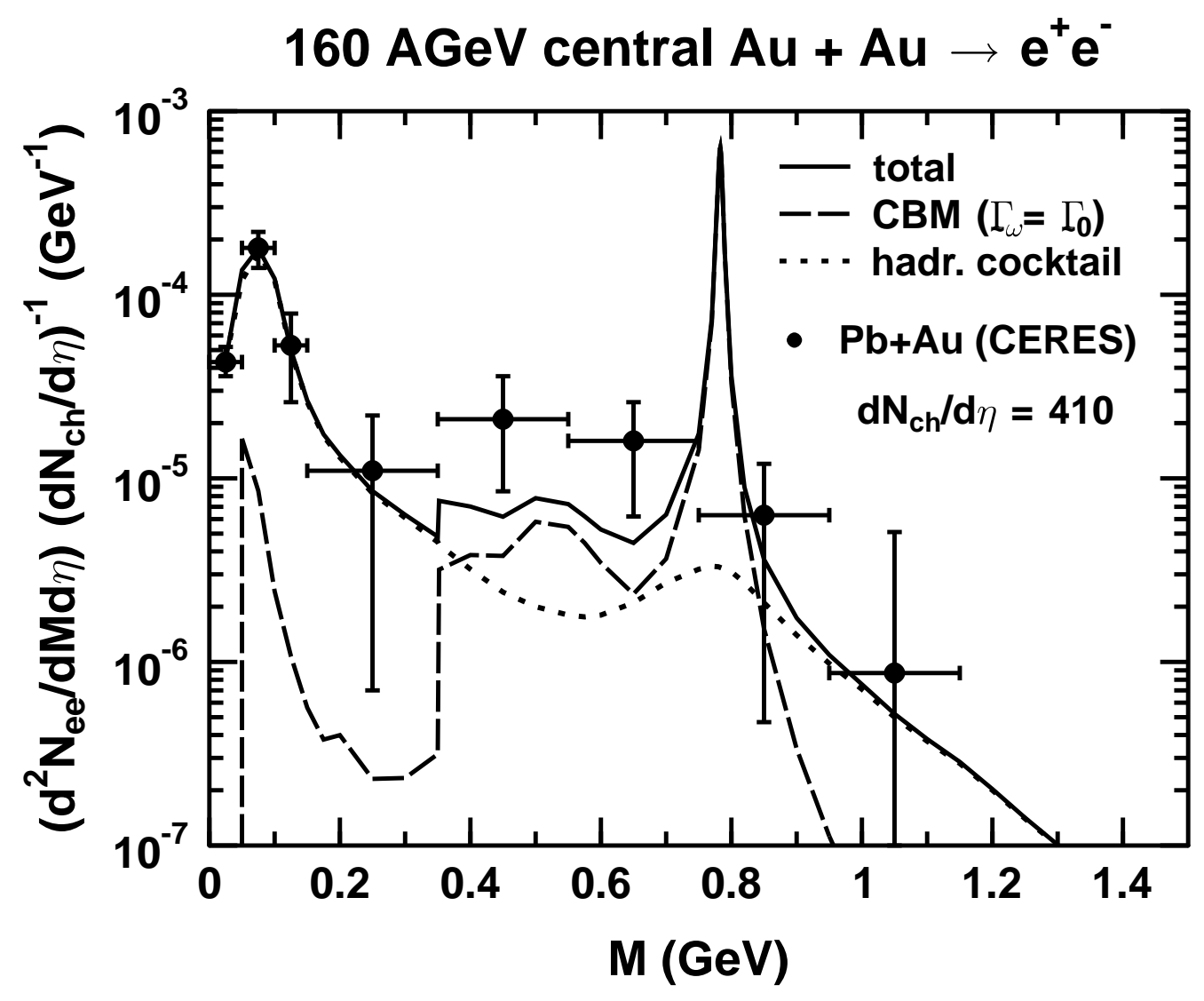




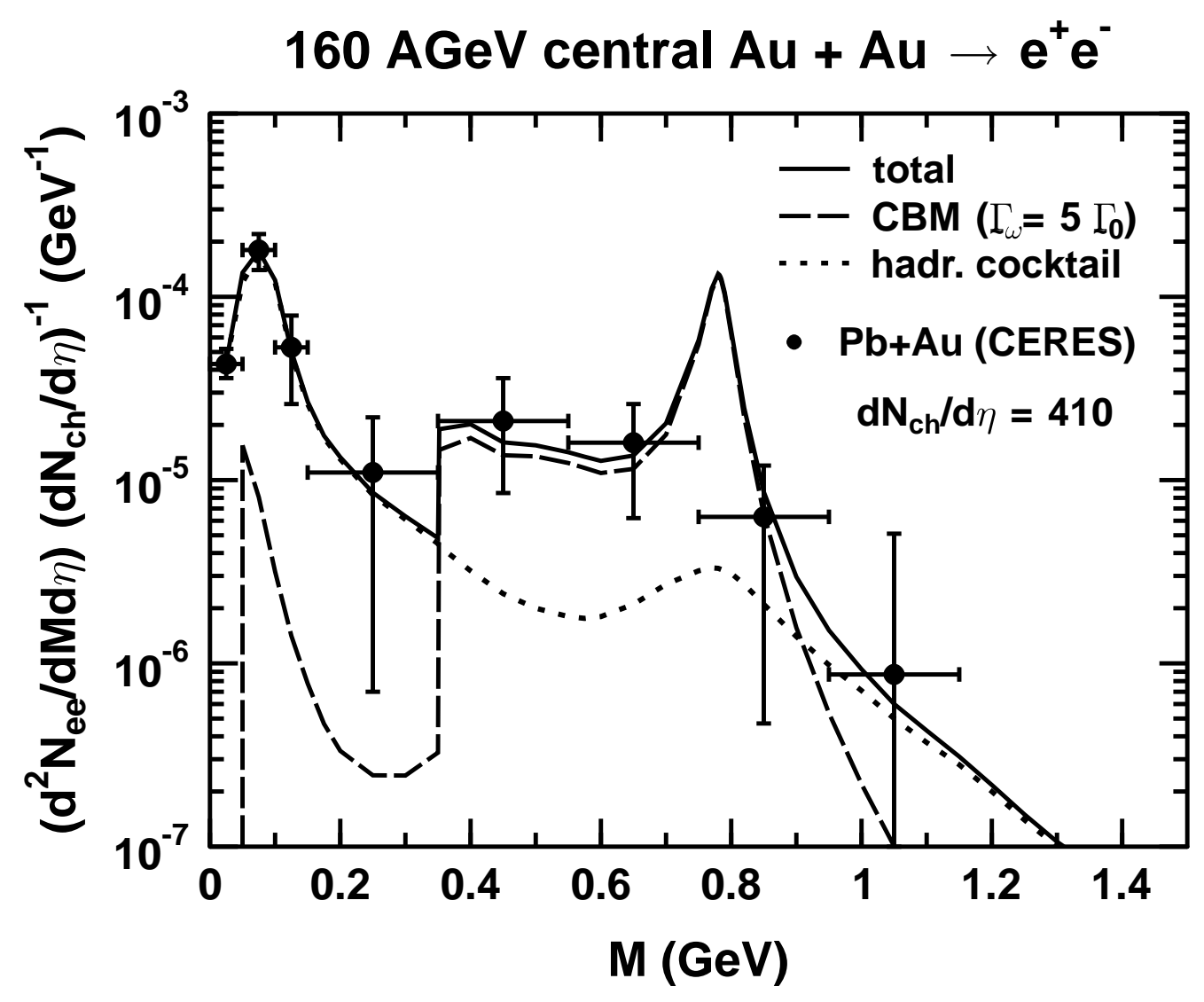




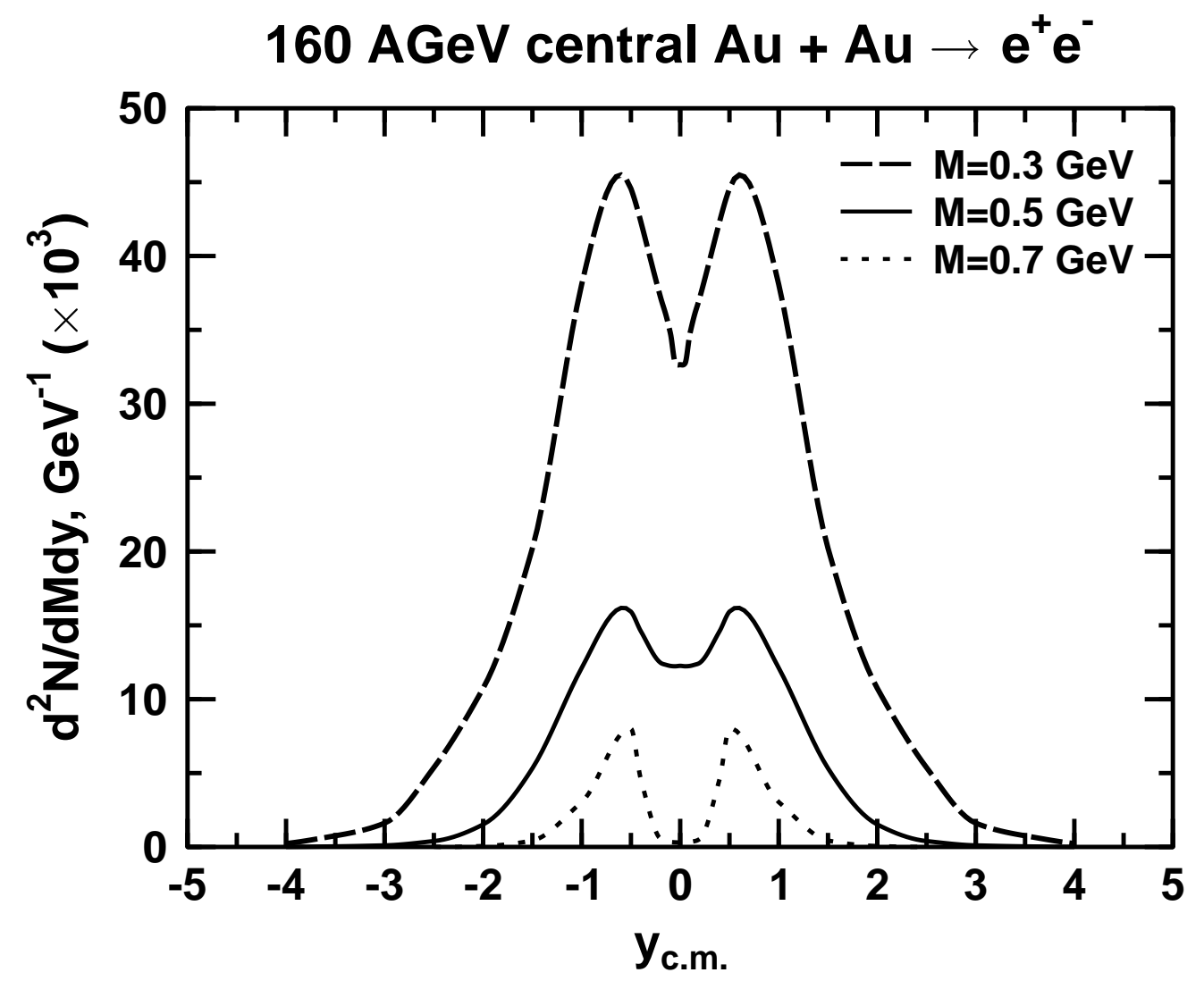

